\title{
QT interval dynamics and heart rate variability preceding a case of cardiac arrest
}

\author{
Jagmeet P Singh, Peter Sleight, Attila Kardos, George Hart
}

\begin{abstract}
A 71 year old man with hypertensive heart disease and chronic renal failure was wearing a Holter monitor when he had a cardiac arrest. He had ventricular fibrillation (VF) and died despite prompt resuscitation. In the 15 minutes preceding the VF there was a sudden increase in heart rate, followed by a brief period of atrial fibrillation leading to ventricular tachycardia, which in turn rapidly degenerated into VF. The QT interval and heart rate variability were studied half hourly over the seven hours preceding the cardiac arrest, using a computerised Holter system. A further detailed analysis was performed over the final hour before the cardiac arrest. An abrupt increase in the steepness of the QT/RR slope, a prolonged QTc, and a reduction in the heart rate variability were observed in the interval that immediately preceded the onset of the terminal rhythm disturbance.
\end{abstract}

(Heart 1997;77:375-377)

Keywords: QT interval variability; heart rate variability; cardiac arrest; left ventricular hypertrophy

Despite the high prevalence of sudden cardiac death, few patients have a cardiac arrest while wearing an ambulatory electrocardiographic recorder, which would enable analysis of the initiating cardiac arrhythmia and the period immediately preceding the arrhythmia. The duration of ventricular repolarisation is poorly explored on the conventional electrocardiogram. Static QT interval measurements from the surface electrocardiogram and their correction for the influence of heart rate using Bazett's formula may be inadequate, since the QT interval is also continually conditioned by changes in the activity of the autonomic nervous system. ${ }^{12}$ We therefore studied for the first time the dynamic behaviour of the QT interval on a beat to beat basis together with heart rate variability, in the period immediately preceding the terminal rhythm.

\section{Case report}

The patient was a 71 year old man with a history of hypertensive heart disease and chronic renal failure who was on maintenance dialysis. Two months previously he had an episode of paroxysmal atrial fibrillation. His medication included digoxin $62.5 \mathrm{mg}$ once a day, ferrous sulphate $200 \mathrm{mg}$ twice a day, and temazepam $10 \mathrm{mg}$ at bedtime.

He was admitted to hospital with a transient ischaemic attack. On physical examination the pulse was regular at 82 beats $/ \mathrm{min}$, blood pressure was $200 / 108 \mathrm{~mm} \mathrm{Hg}$, and the jugular venous pressure was normal. There were no pulmonary abnormalities. The heart sounds were normal; there was a soft ejection systolic murmur over the second right intercostal space. His serum potassium was $5.7 \mathrm{mmol} / \mathrm{l}$. The ECG showed sinus rhythm, left ventricular hypertrophy, and a QS pattern in the inferior leads. On chest $x$ ray there was moderate cardiomegaly, confirmed by echocardiography which showed moderate left ventricular hypertrophy and mild dilatation, but with preserved ventricular function. A few days after admission a Holter monitor was fitted because of repeated episodes of giddiness. Seven hours into this recording he developed atrial fibrillation followed by a polymorphic ventricular tachycardia which rapidly degenerated into ventricular fibrillation, from which he could not be resuscitated (fig 1).

A computerised Holter analysis system was used to measure the QT interval on a beat to beat basis over the seven hours. The Holter tape was replayed at 200 times the recording speed into a programmable waveform analyser (Century Colour Trace Model, Biomedical Systems). The electrocardiogram was digitised at a sampling rate of $400 \mathrm{~Hz}$, with the QRS signals detected by a template matching algorithm. The working algorithm for QT interval measurement involved a rate dependent search window to locate the $T$ wave peak, following which the point with steepest slope along the descending limb of the $T$ wave and area of least variation were identified. The end of the $T$ wave was defined as the point where the maximum negative slope intersected the
Heart Study, 5 Thurber Street, Framingham, MA

Accepted for publication 13 November 1996 
Figure 1 Ambulatory ECG recording showing a normal sinus rhythm, atrial fibrillation $(A F)$ leading to ventricular tachycardia $(V T), V T$ degenerating into ventricular fibrillation (VF).

Figure 2 Half hourly distribution of the electrophysiological parameters assessed preceding the cardiac arrest. SDRR, standard deviation of the consecutive $R R$ intervals over $5 \mathrm{~min}$ segments; $S D A-Q T$, standard deviation of the average of consecutive uncorrected $Q T$ intervals over 5 min segments; $Q T c$, corrected $Q T$ interval; $Q T / R R$, the slope of the regression line between the $Q T$ and $R R$ intervals.

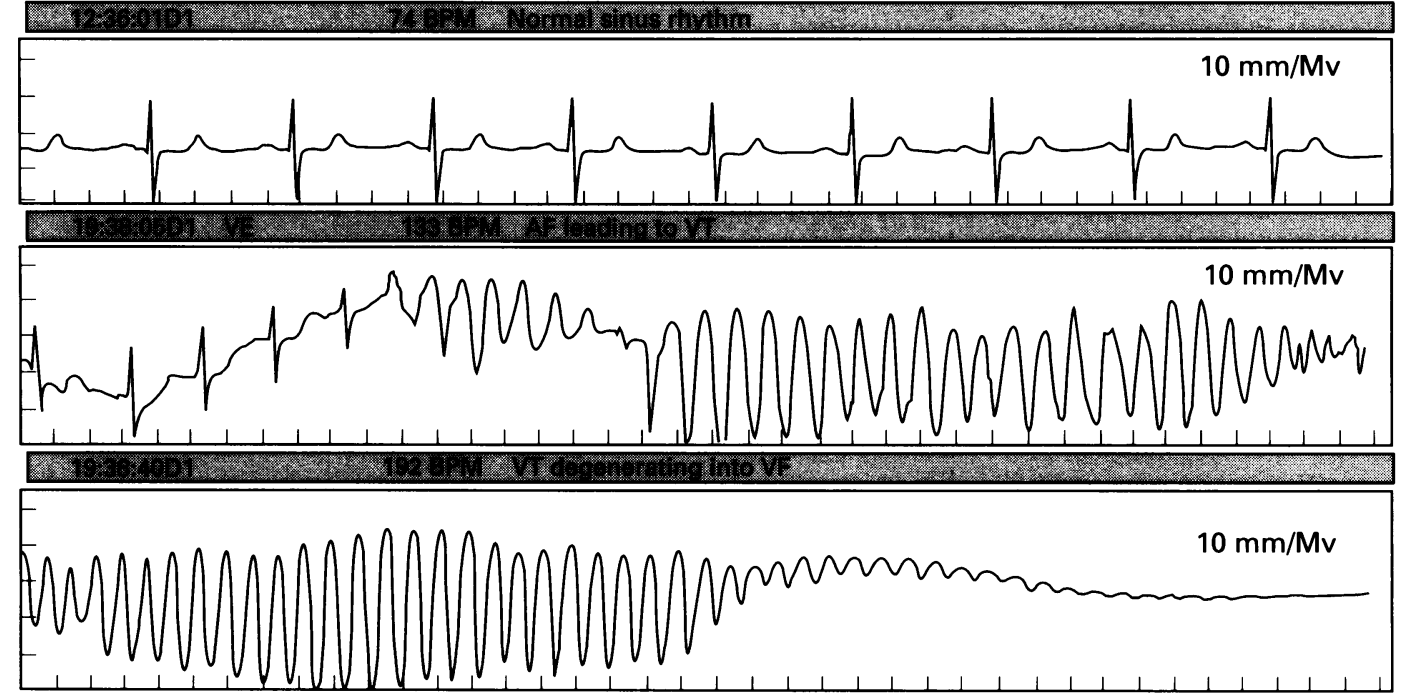

baseline. The QT interval was measured in lead V2 in a semi-automated and interactive manner under operator control. Markers for onset of the QRS and the end of the $T$ wave could be reset by altering the algorithm parameters and rescanning the relevant segments whenever required. A moving average of 10 beats was used to improve signal quality and in turn allow for a more accurate identification of the end of the $T$ wave. Time intervals were
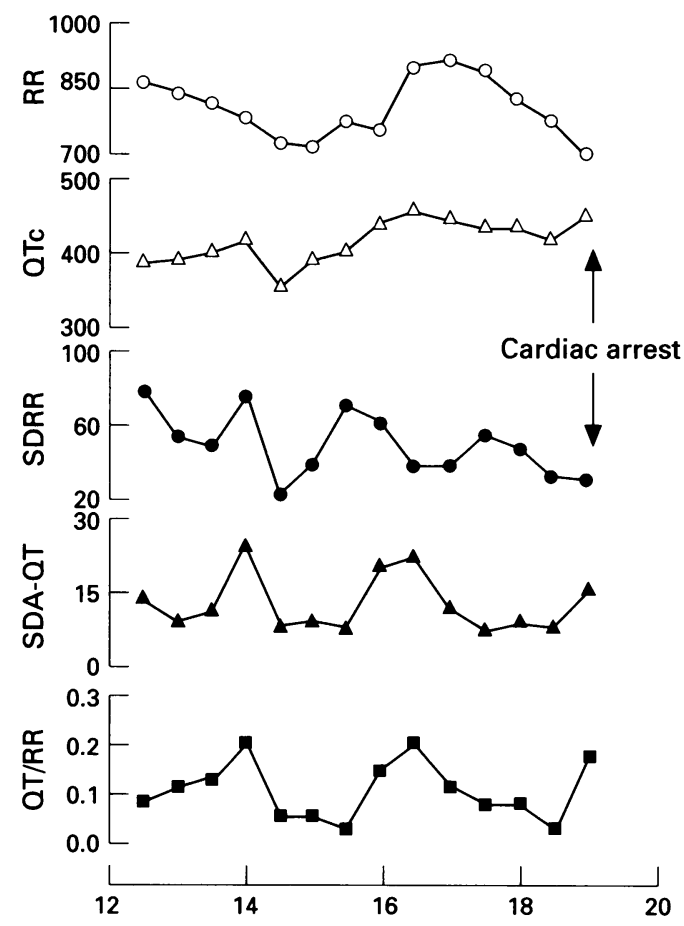

$Q T / R R$ slope and SDRR in the hour preceding the terminal rhythm

\begin{tabular}{lll}
\hline Time $(\mathrm{min})$ & $Q T / R R$ slope & SDRR (ms) \\
\hline-60 & 0.032 & 43.2 \\
-50 & 0.043 & 37.3 \\
-40 & 0.068 & 56.8 \\
-30 & 0.026 & 40.5 \\
-20 & 0.018 & 25.3 \\
-10 & 0.293 & 33.1 \\
\hline
\end{tabular}

SDRR, standard deviation of consecutive RR intervals over 5 min intervals. resolved to $2.5 \mathrm{~ms}$. Artefacts and supraventricular and ventricular ectopic beats were excluded from the analysis. All the measured values were stored in ASCII format and subsequently converted to a binary format, from which all calculations were made.

Rate dependent changes in the QT interval were studied as a function of the QT-RR slope. This was derived from regression analysis of the QT intervals with their paired RR intervals over every half hour segment. QT interval variability was measured as the standard deviation of the average of consecutive uncorrected QT intervals over five minute segments (SDA-QT) - a modification of the parameter suggested by Algra et al. ${ }^{3}$ Heart rate variability was defined as the standard deviation of consecutive $R R$ intervals over successive five minute intervals (SDRR). Segments with excessive noise and ectopic activity were rejected. The segment with atrial fibrillation was excluded from the analysis. The QT and $R R$ interval dynamics were studied on a half hourly basis until the onset of atrial fibrillation, with a further detailed analysis involving 10 minute segments of the last hour preceding the fatal arrhythmia.

Figure 2 illustrates the trend of the half hourly averages of the electrophysiological parameters. The final segment preceding the terminal rhythm showed a prolonged QTc interval (452 ms), a short $R R$ interval (703 ms), a steep rise in the QT/RR slope $(0 \cdot 18)$, a step up in the SDA-QT $(16 \cdot 1 \mathrm{~ms})$, and a fall in the SDRR $(31.7 \mathrm{~ms})$. The table summarises the 10 minute distribution of the SDRR and QT/RR slope in the hour before the terminal rhythm. The SDRR remained consistently low while a marked increase in the QT/RR slope was observed in the last $10 \mathrm{~min}-$ utes before the fatal rhythm disturbance.

\section{Discussion}

This case raises several interesting points about the dynamics of ventricular repolarisation in the period immediately preceding the terminal rhythm disturbance in a subject who had a fatal cardiac arrest. 
The development of malignant ventricular arrhythmias at the time of sudden cardiac death has been attributed to multiple causes. An increased sympathetic nervous system activity has been shown to decrease the ventricular fibrillation threshold. ${ }^{4}$ This is further substantiated by the efficacy of $\beta$ blockers in reducing the incidence of sudden cardiac death after myocardial infarction. ${ }^{5}$ We observed a decreased heart rate variability immediately before the terminal rhythm, which is in agreement with the findings of Martin et al. ${ }^{6}$ This decrease in heart rate variability, along with an increasing heart rate, may possibly reflect increased sympathetic tone. There is also evidence to suggest that atrial fibrillation could be mediated by abnormalities in the autonomic tone. ${ }^{1}$

Since all arrhythmogenic processes involve the action potential, study of the dynamic changes in ventricular repolarisation may be relevant. From the half hourly trend (fig 2), a rise in the QT/RR slope is seen in the final segment preceding the terminal rhythm. A further detailed analysis of the final hour shows that the increase in QT/RR slope is maximal in the 10 minute segment immediately preceding the arrhythmia, suggesting that changes in duration of the ventricular repolarisation for a particular change in the heart rate are more pronounced. This implies that the repolarisation segment is most unstable during this period and could favour the emergence of reentrant arrhythmias. This is supported by an increase in the SDA-QT which is a measure of the temporal dispersion of the QT interval. A particularly interesting feature is that the SDA-QT goes out of phase with the SDRR in the final segment preceding the cardiac arrest; that is, there appears to be an increase in the QT interval variability while the heart rate variability is reduced. The presence of a prolonged QTc in the last segment might favour the emergence of the early afterdepolarisations, especially in the setting of increased sympathetic tone. ${ }^{1}$ The QT/RR slope has been shown to be influenced by the prevailing autonomic tone and blunted by $\beta$ blockers. ${ }^{7} \beta$ blockers might prevent sudden death by reducing the sympathetic tone, thereby blunting the QT/RR slope and in turn stabilising repolarisation.

It is likely that these abnormalities in the electrophysiological parameters may be markers of the vulnerable myocardium with increased sympathetic tone acting as a potential trigger. These ambulatory ECG precursors of sudden death were seen to fluctuate and approached pre-arrest measures at 1400 and 1630 hours (fig 1 ). This suggests that the final event leading to ventricular fibrillation is difficult to predict from these parameters alone and involves a complex interplay of triggers and modulators that act on a vulnerable myocardium. The trends in the electrophysiological parameters, however, seem to suggest that the final lethal arrhythmia results from a chain of events.

Abnormalities have been described in the static $^{8}$ and recently the dynamic behaviour ${ }^{9}$ of the QT interval in left ventricular hypertrophy. One can speculate that the aberrations observed in the dynamic behaviour of the QT interval in the setting of an underlying arrhythmogenic substrate, that is the hypertrophied heart, could precipitate re-entry and triggered arrhythmias. Although no ST segment depression was found preceding the cardiac arrest, it is possible that ischaemia could have been overlooked since only two leads were monitored. A contributory effect of digoxin to the terminal arrhythmia cannot be ruled out. ${ }^{10}$

In summary, a Holter recording in a patient with sudden cardiac death revealed abnormalities in the dynamic behaviour of the repolarisation segment and a reduced heart rate variability immediately preceding the terminal rhythm disturbance.

1 Coumel P. Cardiac arrhythmias and the autonomic nervous system. $\mathcal{F}$ Cardiovasc Electrophysiol 1993;4:338-55.

2 Molnar J, Zhang F, Weiss J, Ehlert FA, Rosenthal JE. Diurnal pattern of QTc interval: how long is prolonged? $\mathcal{f}$ Am Coll Cardiol 1996;27:76-83.

3 Algra A, Tijssen TGP, Roelandt JRTC, Pool J, Lubsen J. QT interval variables from 24 hour electrocardiography and the two year risk of sudden death. Br Heart $\mathcal{F}^{1993}$; 70:43-8.

4 Lown B, Verrier RL, Rabinowitz SH. Neural and psychologic mechanisms and the problem of sudden cardiac death. Am F Cardiol 1977;50:890-901.

5 Peters RW, Muller JE, Goldstein S, Byington R, Freidman $\mathrm{L}$. Propranolol and the morning increase in the frequency of sudden cardiac death (the BHAT study). Am $\mathcal{F}$ Cardiol 1989;63:1518-20.

6 Martin GJ, Magid NM, Myers G, Barnett PS, Schaad JW, Weiss JS, et al. Heart rate variability and sudden death secondary to coronary artery disease during ambulatory electrocardiographic monitoring. Am 7 Cardiol 1987;60: 86-9.

7 Williams EM, Hassan MO, Floras JS, Sleight P. Adaptation of hypertensives to treatment with cardioselective and non-selective beta-blockers. $\mathrm{Br}$ Heart $\mathrm{f}$ 1980;44:473-87.

8 Davey PP, Bateman J, Mulligan IP, Forfar C, Barlow C, Hart G. QT interval dispersion in chronic heart failure and left ventricular hypertrophy: relation to autonomic nervous system and Holter tape abnormalities. Br Heart $\mathcal{F}$ 1994;71:268-73.

9 Singh JP, Marinho MF, Sleight P, Johnston J, Hart G Abnormal rate adaptation of the QT interval in patients with hypertensive left ventricular hypertrophy. Heart 1996;75:26.

10 Leor J, Goldbourt U, Behar S, Boyko V, Reicher Reiss H, Kaplinsky E, et al. Digoxin and mortality in survivors of acute myocardial infarction: observations in patients at low and intermediate risk. The SPRINT Study Group. Secondary Prevention Reinfarction Israeli Nifedipin. Trial. Cardiovasc Drugs Ther 1995;9:609-17. 